\title{
Nuevas perspectivas sobre la pobreza urbana
}

\author{
Gobierno del Estado de México (2003), Pobreza urbana: \\ PERSPECTIVAS GLOBALES, NACIONALES Y LOCALES, GOBIERNO DEL ESTADO \\ de México, Cemapem/Porrúa, México, 395 Pp., \\ ISBN 970-701-368-0.
}

A lo largo de la historia de México, la pobreza ha sido un problema permanente, pero fue sólo hace dos o tres décadas cuando se inició su estudio y se reconoció como un problema estructural de la sociedad mexicana; por ello es importante la aparición de un texto como el de Pobreza urbana: perspectivas globales nacionales y locales. Memoria del Foro Internacional sobre la Pobreza Urbana”, editado por el Gobierno del Estado de México y Editorial Porrúa, y coordinado por el Centro de Estudios sobre Marginación y Pobreza del Estado de México (CEMAPEM).

Este compendio de textos se mira como un libro necesario, y me atrevería a decir, indispensable, en las bibliotecas de todos aquellos interesados por la situación de pobreza en que viven millones de seres humanos. De acuerdo con los datos del Banco Mundial, la pobreza está señalada como el problema mayor de los habitantes del planeta, ya que de una población total de 6,000 millones de habitantes en el mundo, 2,800 millones viven con menos de dos dólares al día, y 1,200 millones con menos de un dólar al día (Kanbur, 2001: vi). Actualmente se reconoce categóricamente que en el mundo la brecha entre ricos y pobres va en aumento. ${ }^{1}$

La propuesta de un compendio de estudios sobre la pobreza urbana es, además de necesaria, inquietante, porque trae al primer plano la realidad muchas veces ignorada de los pobres que habitan las ciudades, escondidos bajo el manto de indicadores promedio que no dejan ver los cinturones de miseria, en los que priva el hacinamiento, la precariedad de las viviendas y del salario, cuando no el crimen y la violencia.

Es un hecho que las grandes metrópolis de los países latinoamericanos enfrentan procesos de pauperización de su población y flujos constantes de inmigrantes que no encuentran condiciones

\footnotetext{
${ }^{1}$ Sondeo mundial mediante encuesta realizada por el Banco Mundial a líderes de opinión de 48 países, Princeton Survey Research Associates, 2003
} 
aceptables de vida en ellas, tanto por la saturación del mercado de trabajo, como por su sobrepoblación. La mayoría en la multitud silenciosa que arriba a las ciudades se amontona en los barrios populares y las ciudades perdidas, y encuentra el modo de subsistir en la economía informal. En ese proceso, las grandes ciudades se han convertido en generadoras de problemas sociales y concentran de forma dramática la pobreza. Ante el desafío de sobrevivir en la ciudad, un número significativo de pobres son incorporados a la búsqueda de recursos para proveer el ingreso y el consumo familiares, las mujeres y los menores se ven obligados a trabajar o a mendigar. A falta de una capacitación formal y por la urgencia de sus necesidades, se incorporan al trabajo en condiciones particularmente adversas y discriminatorias: nacen así los callejeros, los desarraigados, los desposeídos, que muchas veces rompen todo vínculo con la sociedad.

Las estadísticas concernientes a la población urbana con frecuencia no dejan ver la realidad desgarradora de las zonas marginales; son conglomerados que se encuentran fuera de la planeación gubernamental y de toda concepción moderna de los espacios urbanos. Es así como este libro aporta evidencia contundente del incremento de la pobreza urbana en nuestro país y referencias importantes para ubicar los casos de otros países y ciudades en el mundo.

Se puede decir que uno de los méritos de este libro es que presenta una visión de conjunto de los estudios sobre la pobreza en las ciudades, y que integra, en alguna medida, una imagen de la composición social de las concentraciones urbanas de nuestro tiempo; una medida que toma el pulso de los problemas sociales, la cual es simultáneamente un ajuste de cuentas con los males permanentes que aquejan a la sociedad mexicana. El rostro de la pobreza que se traza en este libro es el resultado de la colaboración de especialistas con una reconocida trayectoria en la investigación, que aportan los elementos de juicio para aproximar al lector al conocimiento del fenómeno y de los procesos que se vinculan con la pobreza.

Pobreza urbana: perspectivas globales, nacionales y locales está integrado por un conjunto de artículos que a mi juicio tejen tres hilos o ejes que dibujan el fenómeno de la pobreza urbana.

Un primer eje está encaminado a examinar si la política social ha cumplido con sus objetivos de generar mayor bienestar y disminuir los efectos nocivos de la privación. En este hilo se destaca el fundamentado análisis sobre el incremento de la pobreza urba- 
na, a través del cual Araceli Damián y Julio Boltvinik, reconocidos investigadores en el tema de medición de la pobreza, trazan con mano firme los rasgos de la evolución y características de la pobreza en México. ${ }^{2}$

Otro de los hilos en esa trama dibuja la pobreza como resultado del funcionamiento del mercado y como el producto del sistema económico. Esa óptica nos permite ver la pobreza en el contexto de la acentuación de la orientación neoliberal en el modelo de desarrollo, enfatizando las rupturas del modelo y sus efectos devastadores sobre los pobres. Se abordan en este contexto los impactos de la globalización y la liberación de los mercados sobre los menos favorecidos y los conceptos de vulnerabilidad y exclusión de los sectores de población en condiciones de privación.

Uno de los ejes más importantes que dan sentido al libro se articula desde la perspectiva de la vulneración de los derechos humanos y sociales, es decir, de la medida en que la pobreza es un obstáculo para el ejercicio de los derechos de ciudadanía. Como lo expresa Amartya Sen al considerar que el desarrollo puede ser visto como un proceso de expansión de los derechos y libertades de que disfrutan los individuos, una de las principales fuentes de privación de la libertad es la pobreza, que limita la libertad de los seres humanos para satisfacer sus necesidades de nutrición, para poner fin a enfermedades tratables, para vestir dignamente, para vivir con un mínimo de decoro (Sen, 1999).

En esa línea de reflexión, Díaz Urueta presenta las transformaciones territoriales de las últimas décadas como generadoras de nuevas pautas de segregación y como modeladoras de patrones socioespaciales de exclusión; a su vez, Martha Schteingart pone en relieve las relaciones entre la pobreza y otras problemáticas sociales como las referidas al género, la violencia y el ambiente que vulneran también el ejercicio de los derechos humanos; Jorge Arzate muestra las concepciones cualitativas de la pobreza, y María Angélica Luna Parra pone el acento en los efectos depredadores de la privación sobre la cohesión social.

Varios de los artículos ejercen la crítica a las políticas y a los programas gubernamentales para atender a los pobres, crítica que rechaza cualquier tipo de atenuantes: enjuician, observan, plantean alternativas y señalan las brechas que hay por salvar con el fin de alcanzar un porvenir más justo. Destaco en este rubro los estudios de Carlos M. Vilas sobre pobreza, desigualdad y susten-

\footnotetext{
${ }^{2}$ Los comentarios se integran en una visión de conjunto sobre el texto.
} 
tabilidad democrática en el caso de Argentina, y los de Julio Boltvinik y Araceli Damián, sobre los derechos humanos y la medición oficial de la pobreza en México. Los escenarios, como lo advierte Nelson Arteaga al examinar los criterios de diagnóstico puestos en operación por el gobierno federal para el caso mexicano, son pesimistas; la consideración de la pobreza en la política oficial, colocada al margen de los factores económicos, descontextualiza el problema de sus causas estructurales. Bajo ese escenario es previsible no sólo la permanencia, sino la agudización de la pobreza.

En este calidoscopio que nos permite aproximarnos a las distintas facetas de la pobreza apreciamos una doble virtud, la de la amplitud de la mirada y la de su transparencia; el conjunto de ensayos se organiza como una reflexión centrada en la realidad que vivimos en México, pero incluye también la referencia a los procesos de integración en algunos países latinoamericanos; es el caso del trabajo de Juan Pablo Ferrero, sobre el empobrecimiento y la agudización de la exclusión en Argentina.

Rico en temas y términos, el compendio de textos permite adentrarse en las nociones que articulan la visión de la sociedad sobre la pobreza, e incluso aporta nuevos conceptos y nuevos marcos de análisis, que se despliegan en diversos planos del panorama de las investigaciones sobre este tema. Aparecen, así, conceptos como la sociedad global, la ciudad global y las megaciudades, al lado de los barrios y los ghettos; se profundiza en las distinciones entre la vulnerabilidad, la marginalidad y la exclusión; se distingue y estudia la recomposición de los espacios urbanos en términos de la extensión, profundidad y rasgos particulares de la pobreza; por eso el libro es, más que una compilación, una propuesta múltiple, que obliga a discutir con sus autores y a inquietarnos con sus reflexiones.

A lo largo de los artículos toman cuerpo y son examinados procesos tales como la colonización territorial; es decir, la apropiación que de espacios y tierras públicas realizan millones de pobres urbanos que se mueven fuera de la ley para mantener sus posesiones ilegalmente conseguidas. Esos procesos de "usurpación silenciosa", generadores de formas inéditas del caos, e interpretados muchas veces como un regreso a la barbarie, son en el fondo -como lo afirma Asef Bayat- estrategias simples de supervivencia. En el estudio "Ciudades del Medio Oriente", Bayat considera esta oposición entre la normalidad de la ciudad ordenada frente a la irregularidad de la vida de los pobres urbanos como 
acciones de supervivencia, articulando así un interesante estudio que bien puede ser aplicado a los problemas de asentamientos humanos irregulares en México.

Varios trabajos en el libro cruzan los ejes del análisis económico, con los de la exclusión y la vulneración de los derechos, como el de María Luisa Lourés, sobre los barrios en crisis en Europa, que combina la discusión sobre la exclusión en el ámbito urbano, con los procesos de acentuación del empobrecimiento y las consecuencias de la modernidad de las ciudades; y el ya mencionado de Boltvinik y Damián, quienes a partir de la premisa de que la forma oficial de medir la pobreza desconoce una parte importante de los derechos humanos de los mexicanos, efectúan un recorrido crítico sobre los criterios oficiales de medición de la pobreza, destacando la forma en que esos parámetros dejan fuera de los programas de apoyo a once millones de mexicanos pobres.

La lucha cotidiana de los actores políticos reflejada en el libro es percibida, en buena parte de los estudios, como la forma natural y lógica de hacer la vida. Así, en el conjunto de capítulos contenidos en el compendio salen a la luz los rostros de los inmigrantes, de los refugiados, los desempleados, los vendedores de la calle; esos ocultos protagonistas urbanos que cotidianamente se enfrentan al reto de sobrevivir. Se dibuja en sus páginas el futuro de las ciudades fragmentadas y estigmatizadas por la exclusión, como dice Daniel Hiernaux en su ponencia, marcadas por la violencia, la ilegalidad y la segregación espacial, privativas de los espacios urbanos.

Las características particulares de la pobreza urbana en el Estado de México son abordadas desde distintas perspectivas por Ana Lilia Herrera, Alfonso Iracheta, Laura Mota, Suárez Pareyón y Jesús Enrique Velázquez, Enrique Moreno Sánchez y Juan Carlos Patiño. En el trazo de esos ensayos, no dejan de ser impresionantes las dimensiones de los problemas que afectan a la entidad más poblada del país, los que además de constituir una evidencia apabullante de la pobreza urbana, demandan revertir el sesgo ruralizante de las políticas de atención a la pobreza como formas de intervención que han dejado al margen la atención al crecimiento desmesurado de las zonas metropolitanas de los valles de México y Toluca; poniendo al descubierto a millones de habitantes que carecen de seguridad social, de servicios públicos, de transporte, en áreas donde prevalece una disminuida calidad de vida; si no es que se mueven en condiciones degradantes de pobreza. 
No falta tampoco una dimensión ética necesaria al momento actual, tan desprovisto de iniciativas solidarias que combatan la crudelísima hegemonía del individualismo, la que se condensa en la propuesta sobre el proyecto de carta mundial por el derecho a la ciudad.

Resulta sugerente y motivador penetrar en estos inteligentes estudios que dan cuerpo y sustancia al empobrecimiento y a los pobres urbanos, sumando y enfrentando posiciones que serán sin duda provocadoras del acuerdo y de la controversia. Como se advierte al inicio de estas consideraciones, la pobreza es hoy por hoy el problema más importante en el mundo, y requiere de profundas reflexiones y de nuevas ideas. En este sentido, el libro Pobreza urbana, perspectivas globales, nacionales y locales constituye una valiosa aportación al pensamiento y a la discusión teórica sobre la pobreza, y una iniciativa para mantener la esperanza de que en el futuro veamos nacer una sociedad más humana y solidaria.

\section{Bibliografía}

Kanbur, Ravi (2001), Banco Internacional de Reconstrucción y Fomento, Banco Mundial.

Sen, Amartya (1999), Development as freedom, Nueva York, Alfred A. Knopf Editores.

Gloria Guadarrama gguadarr@cmq.edu.mx 